\title{
Psycho-Oncologie remercie tous les experts qui ont aidé à améliorer la qualité des articles de la revue en 2015
}

(C) Lavoisier SAS 2016

Parmi eux remercions :

Dolorès Albarracin

Jérome Alric

Vincent Angel

Emmanuel Babin

François Baillet

Pierre Baumann

Nicolas Bendrihen

Gilles Bertschy

Olivier Bézy

Diane Boinon

Jessica Bouak

Caroline Doucet

Lionel Dany

Olivier Despres

Catherine Durdux

Martine Derzelle

Sylvie Dolbeault

Ariane Eberl-Marty

Livia Edery

Françoise Elien

Lise Fillon

Cécile Flahault

Pierre Gagnon

\author{
Cécile Glineur \\ Dominique Gros \\ Patrice Guex \\ Didier Jacqmin \\ Philippe Jaury \\ Sylviane Jeanroy-Beretta \\ Laurent Lemaître \\ Joëlle Ligghezzolo \\ Frédéric Limosin \\ Sandrine Limousin \\ Eva Louvet \\ Isabelle Marcoux \\ André Mariage \\ Gilles Marx \\ Thierry Meyer \\ Sabine Noal \\ Georges Noël \\ Nicole Pélicier \\ Agnès Perpère \\ Sylvie Pucheu \\ Edith Salles-Vuillemin \\ Etienne Seigneur
}

